\title{
Synchrotron X-ray microtomography and 3D image analysis of medium density fiberboard (MDF)*
}

\author{
Thomas Walther ${ }^{1, * *}$ and Heiko Thoemen ${ }^{2}$ \\ ${ }^{1}$ Voith Paper GmbH \& Co. KG, Heidenheim, Germany \\ 2 Department of Wood Science, University of Hamburg, \\ Hamburg, Germany \\ ${ }^{*}$ Corresponding author. \\ Voith Paper GmbH \& Co. KG, St. Poeltener Strasse 43, \\ 89518 Heidenheim, Germany \\ Phone: +49-7321-372061 \\ Fax: +49-7321-377102 \\ E-mail: thomas.walther@voith.com
}

\begin{abstract}
The three-dimensional microstructure of a series of laboratory medium density fiberboard (MDF) samples with densities varying from $300 \mathrm{~kg} \mathrm{~m}^{-3}$ to $1000 \mathrm{~kg} \mathrm{~m}^{-3}$ was investigated by synchrotron microtomography (SR $\mu \mathrm{CT}$ ). After reconstruction and filtering of the volume data, an adapted image analysis was used to extract the structural information about the fiber network at different densities. Information was established on the distribution of intra- and inter-fiber voids, cell-wall material, individual fibers, and fiber bundles. Calculations were performed concerning the surface area towards the ambient void spaces, the number of contacts, and the contact area between selected fibers. Furthermore, the resin distribution on the fibers was investigated and characterized by $\mathrm{SR} \mu \mathrm{CT}$ and environmental scanning electron microscopy with stained adhesives. The results presented here allow a new approach to examine the complete wood fiber network of MDFs by automatic image analysis routines.
\end{abstract}

Keywords: adhesive distribution; fiber network; image analysis; medium density fiberboard (MDF); microtomography; three-dimensional structure.

\section{Introduction}

The micro-structure of natural fiber-based materials has a significant effect on most of their physical and mechanical properties. In order to investigate the structureproperty relationship, appropriate measures are needed to determine the material structure on the scale of individual fibers. For wood-based composites, microscopic methods, including light microscopy, scanning electron microscopy, and transmission electron microscopy have been applied extensively in the past to achieve this aim. Examples of such work are given by Kruse and Parameswaran (1978), Parameswaran and Himmelreich

* Dedicated to Prof. Dr. Arno Frühwald on the occasion of his 65 th birthday and his retirement.
(1979), Short (1981), Murmanis et al. (1986a,b), Donaldson and Lomax (1989), Butterfield et al. (1992), and Gillah (2000).

However, such images are only two-dimensional (2D) in nature. Even when considering the substantial depth of field attainable, e.g., by confocal laser scanning microscopy, microscopic techniques do not provide full three-dimensional (3D) images that can be used as the basis for a quantitative analysis of the sample structure. An approach presented by Ramli et al. (2002), who arranged several thin 2D slices to obtain a spatial image of a fiberboard sample, may provide basic information about their 3D structure, but the sample preparation is very labor intensive. Therefore, such an approach based on microscopic images is not widely applicable.

In recent years, X-ray computed microtomography $(\mu \mathrm{CT})$ has developed as a powerful tool to investigate the $3 D$ structure of materials. $\mu C T$ techniques not only generate spatial images as a basis for micro-structural analysis but they also allow a free selection of virtual cuts through the sample in any direction. Consequently, surface artifacts caused by the sample preparation do not derogate the analysis. This is of particular importance for materials that are sensitive towards microscopy preparation, such as medium density fiberboard (MDF).

The limitations of laboratory X-ray beam $\mu \mathrm{CT}$ systems regarding resolution and contrast, when examining wood structures, can be resolved by synchrotron X-ray sources with a focused X-ray beam that allows high-resolution scans with a high contrast as they are needed for displaying and analyzing individual wood fibers.

However, due to the enormous experimental costs, only a small number of investigations by synchrotron $\mu \mathrm{CT}$ have been published in the field of wood science so far. Shaler (1997) and Groom et al. (1999) showed a first qualitative and quantitative analysis of very small MDF samples $\left(1.28 \times 1.09 \times 0.37 \mathrm{~mm}^{3}\right)$ scanned at the National Synchrotron Light Source (USA). Focusing on strand-based wood composites, Sugimori and Lam (1999) performed a quantitative analysis of the macrovoid distribution in such materials. Based on a $\mu \mathrm{CT}$ analysis of low-density wood-based fiberboards, Faessel et al. (2005) and Lux et al. (2006) developed a model of a 3D fibrous network. Similar research was carried out by Holmstad (2004) and Bache-Wiig and Henden (2005), who supplemented the 3D analysis of paper structures by microtomography investigations.

IIIman and Dowd (1999) characterized solid wood by $\mu C T$ and performed 3D scannings of southern yellow pine (Pinus palustris) samples treated with the brown-rot fungus Gloeophyllum trabeus. Ekevad (2004) and Steppe et al. (2004) identified the anatomical structure of wood samples by $\mu \mathrm{CT}$.

Some of these works already include a quantitative analysis of the $\mu \mathrm{CT}$ data. In most cases, however, the 
data analysis is rather qualitative in nature, as the adaptation of existing software routines to the material in question is typically quite elaborate. Moreover, in many cases suitable routines have to be entirely developed from scratch.

The aim of this paper was to develop a method based on automated image analysis and evaluation routines to quantitatively evaluate the micro-structure of wood fiber networks, and to demonstrate the applicability of this method within a wide density range. In addition, the suitability of this method to characterize the resin distribution within a wood fiber network will be assessed and discussed in this paper.

\section{Materials and methods}

\section{Sample preparation}

For the microtomography investigations, laboratory boards were made of untreated softwood fibers (Pinus sylvestris L.) from a conventional MDF plant by means of a computer-controlled miniature hot-press. The boards had a thickness of $5 \mathrm{~mm}$ and a diameter of $100 \mathrm{~mm}$, with target densities of $300 \mathrm{~kg} \mathrm{~m}^{-3}, 500$ $\mathrm{kg} \mathrm{m}^{-3}, 800 \mathrm{~kg} \mathrm{~m}^{-3}$ and $1000 \mathrm{~kg} \mathrm{~m}^{-3}$. The solid resin content of the boards was set to $10 \%$ related to the dry weight of the board. A standard urea formaldehyde resin (BASF Kaurit 350, BASF, Germany) and $1 \%$ ammonium-nitrate as a hardener was used. No additional wax was added. The fibers were treated with the resin mixture in a laboratory blender resulting in an average moisture content $(\mathrm{MC})$ of $11 \%$ related to the total dry weight of the mat.

The press was closed within $10 \mathrm{~s}$ to target thickness, followed by a period of constant thickness of $75 \mathrm{~s}$ to gain a symmetrical density profile with a minimum in the middle layer and a maximum in the surface layers (see Figure 1). Samples of $50 \times 50 \times 5 \mathrm{~mm}^{3}$ were cut out from the center of the samples, and the density profile was measured by a gamma X-ray density scanner (Raytest, Rayscan Isotopenmessgeräte, Berlin, Germany). Small samples were cut out of the center of the square samples with a size of $2 \times 2 \times 5 \mathrm{~mm}^{3}$ for the microtomography measurements. Cutting was done by razorblades to create a smooth surface and to reduce preparation artifacts. The sample edge length of $2 \mathrm{~mm}$ was selected to allow a high resolution with the tomography settings and to gain a sample size large enough for the quantitative analysis of the material structure. Another limiting factor was the amount of data that could be handled by image analysis and computational power.

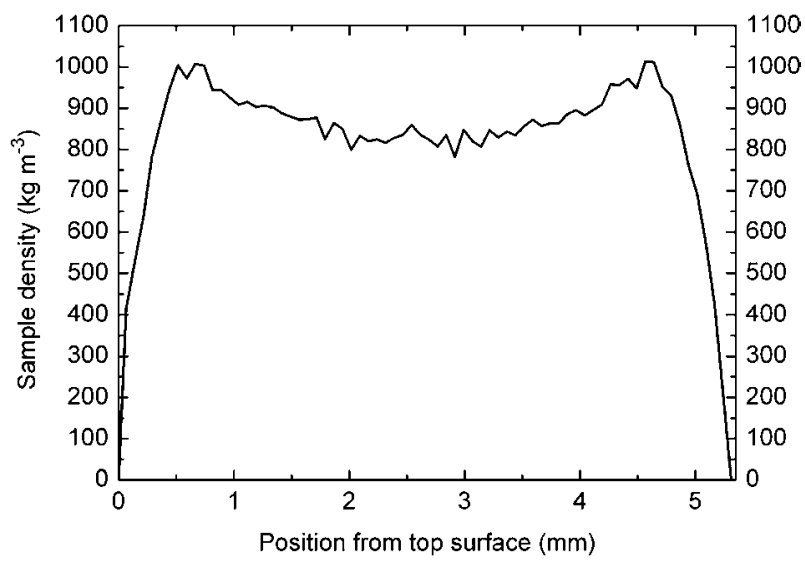

Figure 1 Symmetrical density profile of a MDF sample with a target density of $800 \mathrm{~kg} \mathrm{~m}^{-3}$.

\section{Microtomography}

The SR $\mu \mathrm{CT}$ instrument was a $\mu \mathrm{CT}$-apparatus of the GKSS at beamline BW2 of HASYLAB/DESY (Hamburg, Germany). The advantages of $\mathrm{SR} \mu \mathrm{CT}$ investigations compared to microfocus X-ray tomography are described by Bernhardt et al. (2004). A comprehensive description of the $\mu \mathrm{CT}$ apparatus is given by Beckmann (1998). The samples were glued to the tip of the sample holder and placed inside the tomography apparatus.

The photon energy was adjusted to $12 \mathrm{keV}$. Images were taken at angular positions from $0^{\circ}$ to $179.5^{\circ}$ in $0.25^{\circ}$ steps so that 720 projections with a size of $1536 \times 1024$ pixels each were taken. The monochromatic beam height was $4 \mathrm{~mm}$ with a magnification of 3.94, resulting in an effective pixel size of $2.28 \mu \mathrm{m}$ and a resolution of $3.9 \mu \mathrm{m}$. The full field of view of the CCD camera was $3.51 \times 2.24 \mathrm{~mm}$ (width $\times$ height). Two scans of each sample were taken with some overlap so that a total height of approximately $4 \mathrm{~mm}$ from the top surface layer to almost the bottom surface layer was recorded. Due to the symmetrical density profile (see Figure 1), a scan over the entire thickness of the sample $(5 \mathrm{~mm})$ was not necessary.

A total of 15 samples were scanned, covering the four density levels and including specimens treated with a high absorbing adhesive marker as described below. The reconstruction of the tomography data was carried out at HASYLAB using a filtered back-projection algorithm. The resulting 3D stack of 8-bit TIFF grayscale images with a size of $1536 \times 1536 \times 2028$ voxels was the basic data for the image analysis.

\section{Data processing and image analysis}

The reconstructed grayscale images were further processed for noise elimination and segmentation of the voxels into three groups, i.e., cell-wall material, intra-fiber voids and inter-fiber voids. Intra-fiber voids (the cell lumen) are defined as being completely separated from inter-fiber voids by cell-wall material.

For data processing, sub-volumes defined as small (S) and large (L) with a size of $256 \times 256 \times 256$ voxels and $512 \times$ $512 \times 256$ voxels, respectively, had to be extracted from surface layer and intermediate layer (i.e., between surface and middle layer) positions (Figure 2). The size of the sub-volumes was limited due to memory requirements and computer power; it should be noted that a complete 3D dataset of one MDF sample had a size of 4 GB which was too large to be investigated at once. To save computational time, all routines for the data processing and image analysis were first applied to the small sub-volumes. It was one of the objectives of this work to evaluate whether there is a size effect on the results of the image analysis.

The sub-volumes were analyzed and processed using an image processing library (VIGRA) and adopting the routines to the tomography data by $\mathrm{C}++$. A description of the library is given by Koethe (1999) and Koethe (2000). Due to the voxel edge length of $2.28 \mu \mathrm{m}$, a typical earlywood tracheid wall is only one or two voxels thick. Preliminary tests showed that, under such conditions, simple morphological operations would damage too many fibers and prevent further segmentation. Instead, noise and systematic errors were removed by setting a high threshold against the noise and then a low threshold in the vicinity of those voxels which were above the first threshold. This approach was based on the assumption that all fibers have to be physically connected in order to form a solid material, whereas noise appears randomly throughout the image. By this sequence, a complete segmentation of the data into cell-wall material and air regions was possible.

To further separate the air regions into intra-fiber voids and inter-fiber voids, a region-based approach was applied. First, small holes in the fiber cell walls were closed by morphological operators. Such holes may occur naturally as pits, as a result of 


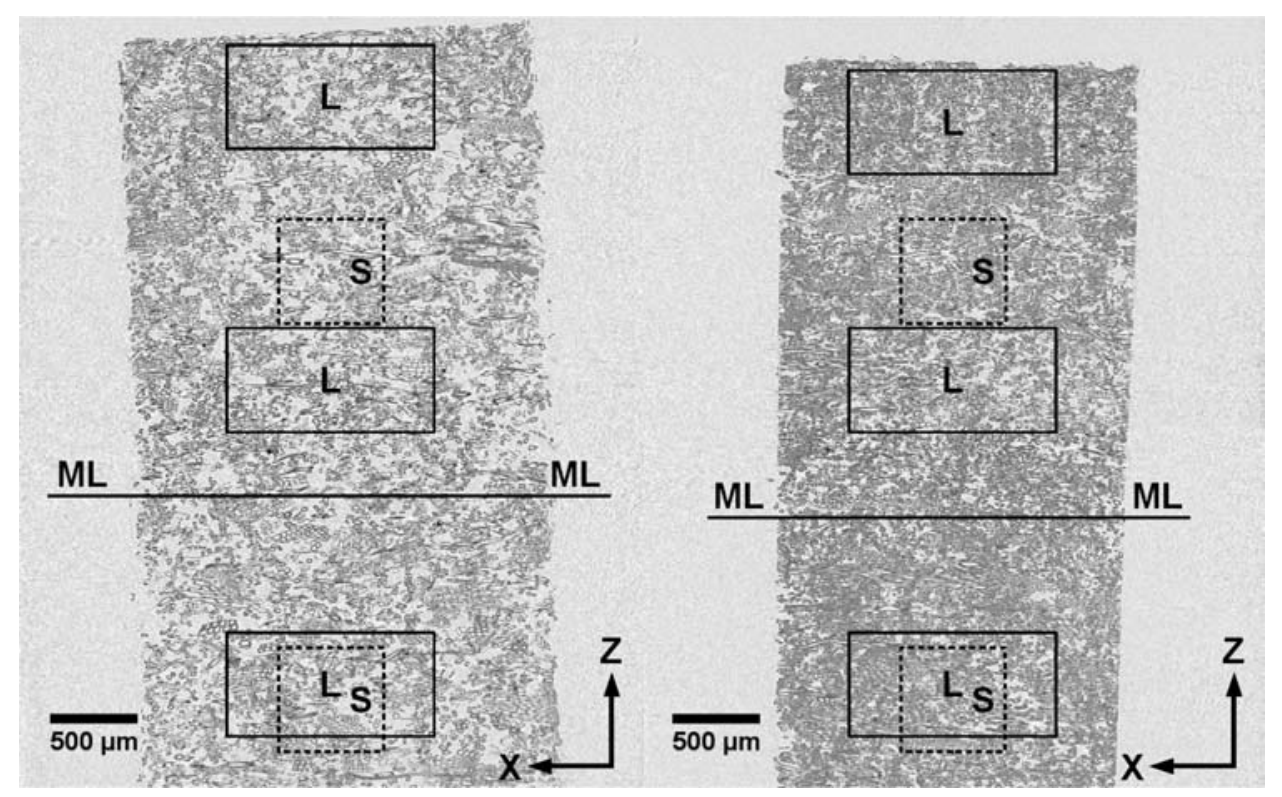

Figure 2 Virtual cross-section of the $\mu \mathrm{CT}$ data of two MDF samples with the marked middle layer (ML) and the positions of the sub-volumes ( $\mathrm{S}=256^{3}$ voxels and $\mathrm{L}=512 \times 512 \times 256$ voxels). Target board density: $500 \mathrm{~kg} \mathrm{~m}^{-3}$ (left) and $800 \mathrm{~kg} \mathrm{~m}^{-3}$ (right).

fiber damage during processing, or as artifacts of the measurement or data processing procedures. The criterion to distinguish intra-fiber voids (cell lumen) from inter-fiber voids was that intrafiber voids are completely enclosed within cell-wall voxels, and that they have a thin, elongated shape. By such definition not all inter-fiber voids are necessarily connected to the ambient air, particularly when coming to higher densities.

The identification of individual fibers was now possible by assigning the nearest surrounding cell-wall material to each individual intra-fiber void. By a combination of a Euclidian distance transformation and seeded region growing, the labels of the individual intra-fiber voids were assigned to the surrounding cell-wall material voxels to establish a so-called Voronoi diagram. A maximum distance for the seeded region growing algorithm of $14 \mu \mathrm{m}$ ( 6 voxels) was found to work best. However, due to some remaining holes in the fiber surface, fiber fragments and noise artifacts, there were still some minor deficiencies in the segmentation and labeling.

The individually marked fibers were now evaluated concerning their orientation, surface areas, and contact areas. The fiber orientation was determined by principal component analysis of the coordinates of all voxels of the intra-fiber void resulting in a covariance matrix. Two calculations were performed: (1) the eigenvector belonging to the largest eigenvalue represents the main direction of the intra-fiber void, and (2) the angle between the eigenvector and the original axes of the volume.

The volume percentage of each segmented component in the MDF sample volume was calculated by counting the voxels of the three regions. To obtain an approximation of the $3 \mathrm{D}$ surface of the cell-wall material in contact with inter-fiber or intra-fiber voids, respectively, a marching cubes algorithm was applied. In addition, a neighborhood analysis was conducted to detect fiber bundles by investigating the position and alignment of the fibers to each other. By examining the contact surfaces between two fibers and by assuming that the contact surface was at least $10^{4} \mu \mathrm{m}^{2}$, fiber bundles were detected. Newly found connected fibers were then added to an already existing fiber bundle or a new bundle was created.

Walther et al. (2006) have described the 3D image processing in detail, including those operations for identifying individual fibers, fiber bundles, surfaces, and contact areas.

\section{Adhesive detection}

Different microscopic techniques to detect adhesives on wood fibers have been applied in the past, e.g., by Youngquist et al. (1987), Donaldson and Lomax (1989), Kamke et al. (2002), Loxton et al. (2003), Xie et al. (2004), and Grigsby et al. (2005). The present paper focused on SR $\mathrm{CCT}$. However, the adhesive cannot be distinguished within the fiberboard sample from the cellwall material by $\mathrm{SR} \mu \mathrm{CT}$, as both matters have similar absorption characteristics. Therefore, to show the adhesive distribution on the fibers and in the solidified sample, the urea formaldehyde resin of some samples was stained with either barium sulfate $\left(\mathrm{BaSO}_{4}\right)$ or iodine (as a component of the contrast agent Ultravist $^{\circledR} 300$, Schering AG, Berlin, Germany) before application. The high absorption of the staining substance causes a pronounced contrast between the stained adhesive and the cell-wall material. The iodine as a water-soluble substance is evenly distributed within the resin solution and probably particularly evaporates during hot-pressing, whereas the barium sulfate as a waterinsoluble substance is thought to be not influenced by the hotpressing process and keeping its position during resin curing. Preliminary investigations revealed that adding $1 \%$ staining substance related to the dry weight of the adhesive was sufficient for a good distribution within the adhesive. It was decided to use both staining substances to see possible differences in distribution.

Sample preparation and SR $\mu \mathrm{CT}$ scanning was performed as described above for the unstained samples. As a control, the resin distribution on the fibers was visualized by an environmental scanning electron microscope (ESEM Type 600 F, FEl, Germany) in backscatter electron detection mode (BSE).

\section{Results and discussion}

\section{MDF tomography data}

The tomography data of the 15 MDF samples were further processed and subjected to image analysis. Two virtual cross-sections in $x, z$-direction are presented in Figure 2. The reconstructed image data displayed are 
without any further processing. The individual fibers and the voids inside and outside of the fibers are clearly visible. The density profile is not visible in Figure 2 in the case of small numbers of slices, but by analyzing the whole data the density profile in z-direction can be developed. The fiber cell-wall thickness of $3 \mu \mathrm{m}$ to $5 \mu \mathrm{m}$ was close to the resolution limit of the microtomography system to derive structural parameters.

The position of the middle layer in the sample displayed in Figure 2 is denoted with $\mathrm{ML}$ and the subvolume areas $S$ and $L$ are outlined. Due to an average fiber length of $3 \mathrm{~mm}$ to $5 \mathrm{~mm}$ of the pine fibers, most of the fibers in the sub-volumes had their starting and ending points outside of the selected area which made the identification of individual fibers easier. A comparison of the image analysis results computed for the two subvolume sizes did not reveal significant difference between them. It can be concluded that the small size is sufficient for the image analysis. Thus, it appears to be justified to include both types of sub-volumes into the presentation and analysis of the results. Findings by Rolland du Roscoat et al. (2007) and Lux et al. (2006) for the representative elementary volume of tomography data of paper were taken into consideration. Furthermore, the results of the permeability and thermal conductivity experiments and simulation presented by Thoemen et al. (2008) showed that the chosen sub-volume size was representative.

The samples have a special cross-sectional density profile. Thus, the sub-volumes close to the middle layer have a lower density than those near the surfaces. In combination with the four target densities, the choice of different positions provided results for a wide range of density levels. The positions of the sub-volumes somewhat inside the samples were chosen to avoid the detrimental influence of surface artifacts due to sample preparation. The total number of sub-volumes for further processing was limited to five per MDF sample because of the long processing time and the limited computer power available for image analysis.

\section{Volume and surface calculations}

Figure $3 a$ shows the distribution of the relative number of voxels per sub-volume, i.e., inter- and intra-fiber voids and cell-wall material. Displayed are the distributions of all 26 sub-volumes $\left(256^{3}\right.$ and $512^{2} \times 256$ voxels, respectively). The average density of each sub-volume is based on $1530 \mathrm{~kg} \mathrm{~m}^{-3}$ density of the cell wall. The MC of the samples during scanning was below $4 \%$. The data obtained by calculation were higher than those obtained by the Raytest density scanner in the section of the selected sub-volume. This density increase may be explained as follows: (1) new cell-wall voxels are generated by the image analysis procedure used to close the artificial holes and pits in the cell walls. (2) The limited resolution of the tomography data contributes to the gain of cell-wall voxels in combination with the threshold chosen for segmenting cell-wall material from air regions. A detailed description of the artificial creation of cell-wall material is given by Walther et al. (2006). (3) Density deviations may be caused by the random local density variations in the $x, y$-plane of the laboratory board.
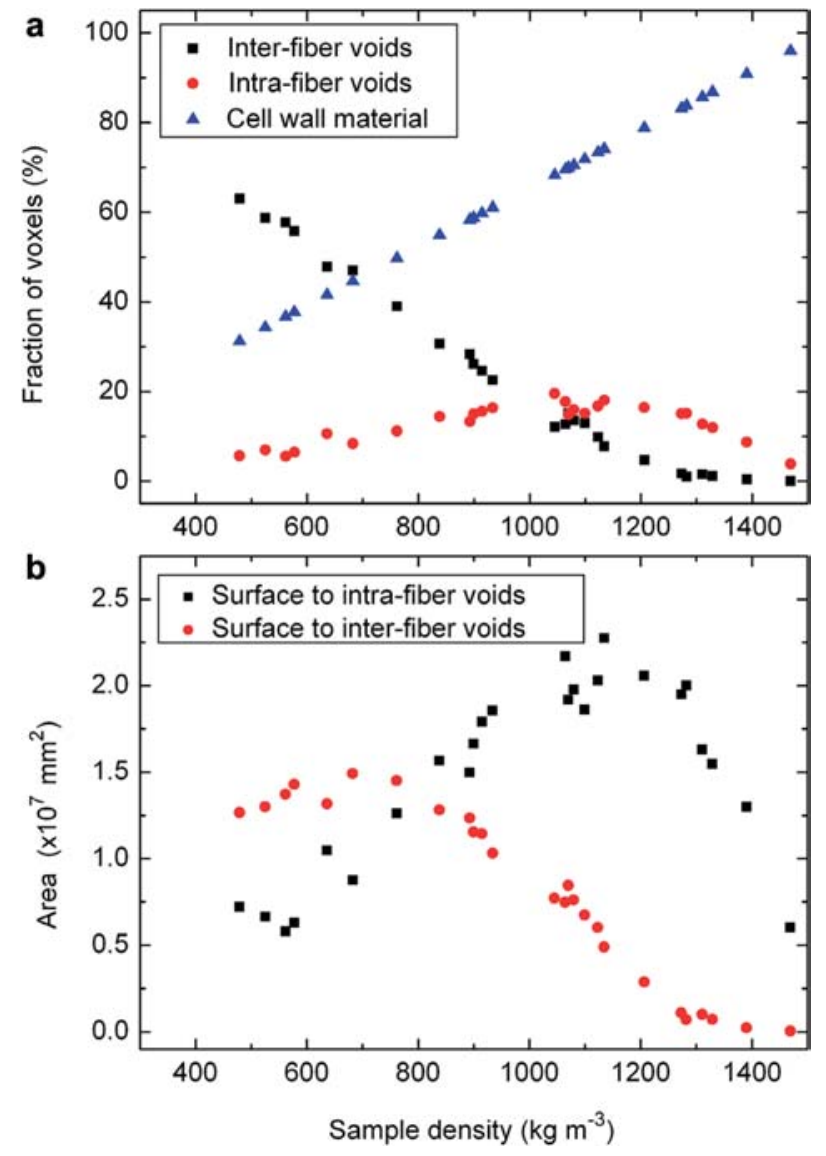

Figure 3 Data as a function of density. (a) Relative number of voxels per sub-volume belonging to cell-wall material, intra- and inter-fiber voids. (b) Area of fiber surface in contact with intraand inter-fiber voids.

The separation of the fiber lumina, the so-called intrafiber voids, seems to be a promising way to find out about structural changes within the fiber network during its compression. Classical approaches for evaluating the lumen volume by mercury porosimetry and the bottleneck effect of the pits (Yamauchi 2008), or image analysis of cross-sections (Niskanen and Rajatora 2002; Reme et al. 2002) are useful when the fibers are intact and neither of the ends are open. However, a dense fiber network might also have enclosed inter-fiber voids that are falsely detected as lumina when applying the classical approaches. The 3D image analysis - that also includes information about the shape of the void and that searches for the surrounding cell-wall material - seems to be a good way to minimize the false allocation of voids. Nevertheless, the identification of single fibers in the tomography data is possible by allocating the right lumen to the surrounding cell-wall material. Furthermore, the sum of the intra- and inter-fiber voids for a given sub-volume density gives an estimation of the porosity of the MDF at this level. A deviation in the allocation of the void spaces at higher densities can be expected but not quantified.

An effect of the beginning total collapse of the fibrous network can be observed in Figure $3 a$ at a density of $1100 \mathrm{~kg} \mathrm{~m}^{-3}$. Insight into the initiation of total collapse of the fibers themselves under higher pressures is gained from measurements by van Houts et al. (2003) for the viscoelastic behavior of wood fibers in MDF as well as 
from SEM pictures taken from the cross-sections of the laboratory MDF boards. This effect depends on the material conditions during hot-pressing, i.e., temperature, MC, and stress level. The collapse of the fibers increases the surface area available for bonding the fibers, and thus increases the internal bond strength. This effect is desired and well understood for papermaking (Kallmes and Eckert 1964).

Fiber segmentation allowed for calculation of the inner and outer surface of the fibers as a function of density (Figure $3 b$ ). The contact surface of the cell-wall material to the surrounding intra- and inter-fiber voids was calculated by a marching cubes algorithm, which approximates the 3D surface of the fibers based on triangle models. The maximum free surface of the fibers to the inter-fiber voids was found at a density of $750 \mathrm{~kg} \mathrm{~m}^{-3}$. This density corresponds with the point where an equal amount of cell-wall material and air are present in the material. The fiber surface area in contact with the intrafiber voids exhibited a maximum around $1100 \mathrm{~kg} \mathrm{~m}^{-3}$, which indicates the point when the total collapse of the fiber network begins due to the severe densification. It can be assumed that only a minor part of the adhesive on the fiber surface is available for bonding up to a density of $750 \mathrm{~kg} \mathrm{~m}^{-3}$. Most of the adhesive cures are without any contact with two adjacent fibers.

\section{Fiber identification}

The whole network within the sub-volume was allocated by allocating each intra-fiber void to the surrounding cell-wall material and identifying the single fibers. The identification of the single fibers was visualized by five different colors and assigning one color to one fiber beginning with the first labeled fiber in the sub-volume (Figure 4).

Furthermore, the detection of fiber bundles was possible by using the neighborhood graph of each detected fiber to find fibers nearby that share a large border. Another effect was that the contact area between the fibers was calculated to obtain an approximate assumption about the bonded area. Selected fibers within the $256^{3}$ voxel sub-volumes with average densities between 300 and $500 \mathrm{~kg} \mathrm{~m}^{-3}$ were found to have an average contact area of $390 \mu \mathrm{m}^{2}$ (SD $\left.80 \mu \mathrm{m}^{2}\right)$. The average number

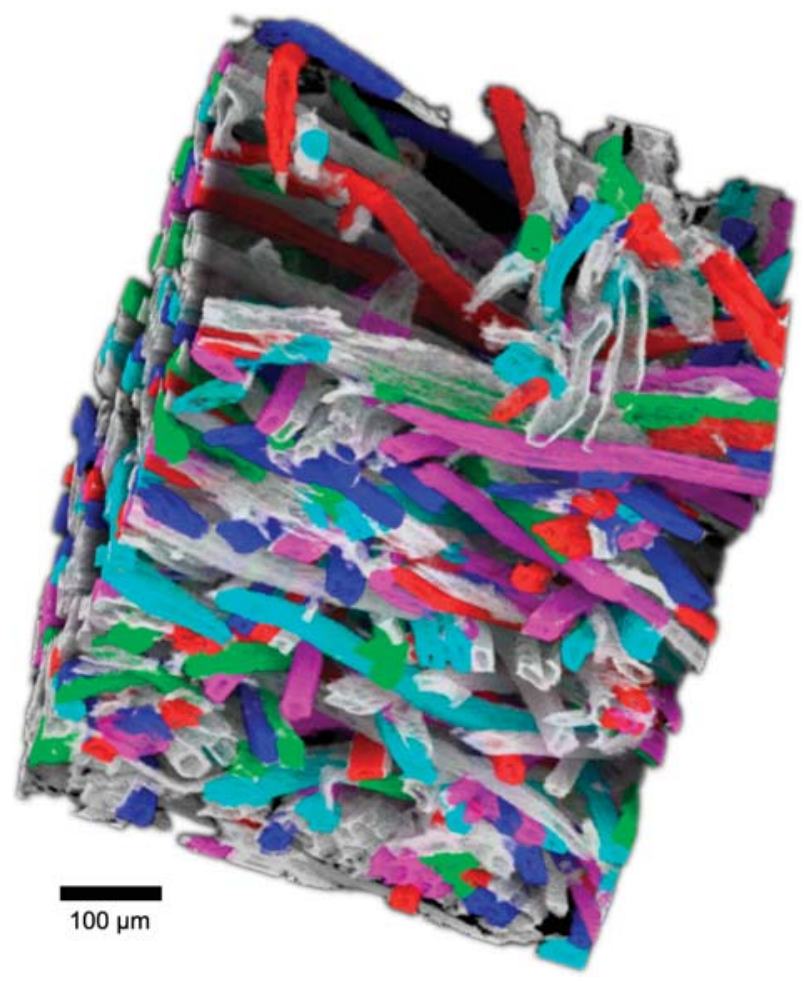

Figure 4 Example of a rendered MDF $256^{3}$ voxel sub-volume with a target density of $300 \mathrm{~kg} \mathrm{~m}^{-3}$ with individually marked fibers.

of contacts per fiber was 16 (SD 3). The analysis was limited to samples with densities below $800 \mathrm{~kg} \mathrm{~m}^{-3}$ because of the resolution of the tomography data and the level of densification.

\section{Adhesive distribution}

ESEM Backscatter electron detection observations made with stained adhesives indicate that the fiber surface was not completely covered with adhesive after blending (Walther 2006). It was found that the barium sulfate particles agglomerated, and an evaluation of the adhesive distribution was not possible. In contrast to this, the iodine-stained adhesive showed a uniform distribution of the staining substance in ESEM BSE mode (Figure 5).

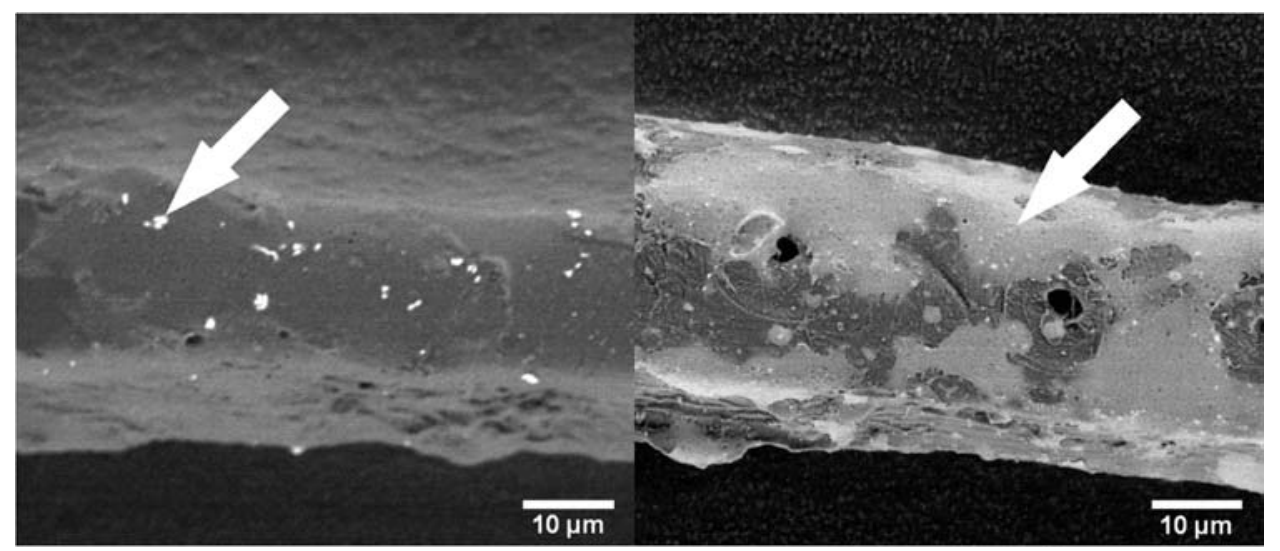

Figure 5 Surface of drum blended single fibers with stained adhesive $(4000 \times)$ in ESEM BSE-mode; left: barium sulfate-stained (arrow indicates barium sulfate agglomeration), right: iodine-stained (arrow indicates iodine-stained adhesive). 


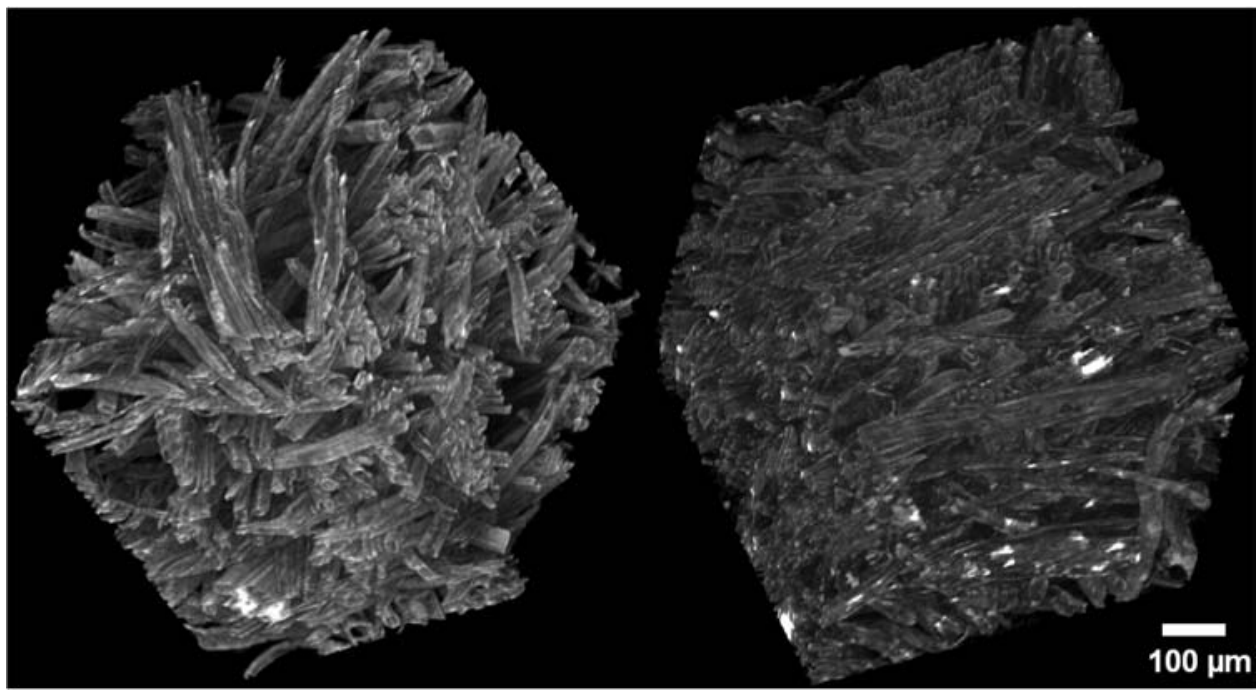

Figure $6 \mu \mathrm{CT}$ images of iodine (left) and barium sulfate (right) -stained MDF $256^{3}$ voxel sub-volumes. The enhanced contrast is due to the iodine-stained adhesive.

The $\mu \mathrm{CT}$ sub-volumes displayed in Figure 6 clearly show the high absorption contrast of the barium sulfate particles, whereas the iodine-stained sample shows an enhanced contrast of the complete network. The resolution of $2.28 \mu \mathrm{m}$ seems to be too low to separate the stained adhesive ( $<1 \mu \mathrm{m}$ in thickness) on the fibers from the cell-wall material. The image analysis of the barium sulfate-stained samples provided insight into the agglomeration of the barium particles. Due to the average barium sulfate particle size of $1 \mu \mathrm{m}$, not all particles were detected. Wang et al. (2007) stained wood plastic composites with Gold nano-particles and found similar results.

\section{Conclusions}

The 3D image analysis of random fiber networks made from wood fibers was successful, and individual fibers have been identified. Standard image analysis routines were adapted to a material consisting of fibers with intrafiber voids inside that tends to collapse under high pressure and which leads to a dense structure. This approach is promising and suited for automatic characterization of the complete structure of MDF. The routines developed worked within a wide density range. However, in the range of high densities of the fiber network the reliability of correct identification of individual fibers decreases. This phenomenon has already been observed in the analysis of tomography data of paper structures (Holmstad 2004). Another disturbing factor is the presence of small noise artifacts despite the previous segmentation and filtering process.

The staining of the adhesive demonstrated that the resin distribution can be visualized by a standard $\mu \mathrm{CT}$ equipment and SEM/ESEM. However, the resolution of the $\mu \mathrm{CT}$ is still not high enough to separate the stained adhesive from the fiber. It is likely, on the other hand, that future developments in X-ray tomography will allow a higher resolution and a good contrast to visualize the bonding network between the fibers and fiber bundles.
Nevertheless, an X-ray absorbing marking substance linking with the adhesive without influencing the adhesive curing will be helpful to conduct a complete 3D adhesive distribution analysis. Research done with fluorescence substances (Loxton et al. 2003; Grigsby et al. 2005) seems to be promising, but it cannot cover the whole 3D structure of even dense MDF samples.

The 3D evaluation of the three components cell-wall material, intra- and inter-fiber voids at different density levels allows the calculation of the permeability and thermal conductivity (Thoemen et al. 2008) and can also be used for research on the mechanical and mechanosorptive behavior of MDF.

\section{Acknowledgements}

The authors gratefully acknowledge the financial support of the Arthur and Aenne Feindt Foundation (Hamburg, Germany) and Tilman Donath and Felix Beckmann from the GKSS at HASYLAB and Hans Meine and Kasim Terzic of the Kognitive Systems Group of the Department of Computer Science, Hamburg University, for their contribution to this research.

\section{References}

Bache-Wiig, J., Henden, P.C. (2005) Individual fiber segmentation of three-dimensional microtomograms of paper and fiber-reinforced composite materials. MSc thesis, Norwegian University of Science and Technology, Trondheim, Norway.

Beckmann, F. (1998) Entwicklung, Aufbau und Anwendung eines Verfahrens der Phasenkontrast-Mikrotomographie mit Röntgen-Synchrotronstrahlung. PhD thesis, University of Dortmund, Dortmund, Germany.

Bernhardt, R., Scharnweber, D., Mueller, B., Thurner, P., Schliephake, H., Wyss, P., Beckmann, F., Goebbels, J., Worch, H. (2004) Comparison of microfocus- and synchrotron X-ray tomography for the analysis of osteointegration around Ti6Al4V-Implants. Eur. Cell. Mater. 7:42-51.

Butterfield, B., Chapman, K., Christie, L., Dickson, A. (1992) Ultrastructural characteristics of failure surfaces in medium density fiberboard. For. Prod. J. 42:55-60. 
Donaldson, L.A., Lomax, T.D. (1989) Adhesive/fibre interaction in medium density fibreboard. Wood Sci. Technol. 23:371-380.

Ekevad, M. (2004) Method to compute fiber directions in wood from computed tomography images. J. Wood Sci. 50:41-46.

Faessel, M., Delisée, C., Bos, F., Castéra, P. (2005) 3D modeling of random cellulosic fibrous networks based on X-ray tomography and image analysis. Compos. Sci. Technol. 65:19311940.

Gillah, P.R. (2000) The influence of novel forming techniques on the properties of medium density fibreboard. PhD thesis, Brunel University, London, UK.

Grigsby, W.J., Thumm, A., Kamke, F.A. (2005) Determination of resin distribution and coverage in MDF by fiber staining. Wood Fiber Sci. 37:258-269.

Groom, L., Mott, L., Shaler, S.M. (1999) Relationship between fiber furnish properties and the structural performance of MDF. In: Proceedings of the 33rd International Particleboard/ Composite Materials Symposium, Pullman, Washington, USA. pp. $89-100$.

Holmstad, R. (2004) Methods for paper structure characterization by means of image analysis. PhD thesis, Norwegian University of Science and Technology, Trondheim, Norway.

IIIman, B., Dowd, B. (1999) High resolution microtomography for density and spatial information about wood structures. In: Proceedings of SPIE's 44th Annual Meeting and Exhibition, Denver, Colorado, USA. p. 7.

Kallmes, O., Eckert, C. (1964) The structure of paper. VII. The Application of the relative bonded area concept to paper evaluation. TAPPI J. 47:540-548.

Kamke, F.A., Scott, K.A., Smith, R.E. (2002) Measurement of resin distribution on MDF fiber: a mill trial. In: Proceedings of the 6th Pacific Rim Bio-Based Composites Symposium, Portland, Oregon, USA. pp. 86-93.

Koethe, U. (1999) Reusable software in computer vision. In: Handbook of Computer Vision and Applications, Volume 3: Systems and Applications. Eds. Jaehne, B., Haussecker, H., Geissler, P. Academic Press, San Diego, CA. pp. 103-132.

Koethe, U. (2000) STL-style generic programming with images. C + + Report magazine 12:24-30.

Kruse, J., Parameswaran, N. (1978) Mikrotechnologische Untersuchungen an Rindenplatten. Holz Roh Werkst. 36:225-233.

Loxton, C., Thumm, A., Grigsby, W.J., Adams, T.A., Ede, R.M. (2003) Resin distribution in medium density fiberboard. Quantification of UF resin distribution on blowline- and dryblended MDF fiber and panels. Wood Fiber Sci. 35:370-380.

Lux, J., Delisée, C., Thibault, X. (2006) 3D characterization of wood based fibrous materials: an application. Image Anal. Stereol. 25:25-35.

Murmanis, L., Myers, G.C., Youngquist, J.A. (1986a) Fluorescence microscopy of hardboards. Wood Fiber Sci. 18:212219.

Murmanis, L., Youngquist, J.A., Myers, G.C. (1986b) Electron microscopy study on hardboards. Wood Fiber Sci. 18:369375.

Niskanen, K., Rajatora, H. (2002) Statistical geometry of paper cross-sections. J. Pulp Pap. Sci. 28:228-233.
Parameswaran, N., Himmelreich, M. (1979) Mikrotechnologische Untersuchungen an Sulfitablauge-gebundenen Holzwerkstoffen. Holz Roh Werkst. 37:57-64.

Ramli, R., Shaler, S., Jamaludin, M.R. (2002) Microstructure of medium density fibreboard from oil palm empty fruit bunch fibre. J. Oil Palm Res. 14:35-44.

Reme, P.A., Johnsen, P.O., Helle, T. (2002) Assessment of fibre transverse dimensions using SEM and image analysis. J. Pulp Pap. Sci. 28:122-128.

Rolland du Roscoat, S., Decain, M., Geindreau, C., Thibault, X., Bloch, J.-F. (2007) Simulation of paper physical properties based on synchrotron X-ray microtomography. In: Proceedings of the 61st Appita Annual Conference and Exhibition, Gold Coast, Australia. pp. 65-70.

Shaler, S.M. (1997) Microtomography of wood composite microstructure. In: Proceedings of the First European Panel Products Symposium, Bangor, Great Britain. p. 28.

Short, P.H. (1981) Scanning electron microscopy of $-60 /+80$ mesh fiberboard furnish. Wood Sci. 14:32-40.

Steppe, K., Cnudde, V., Gerad, C., Lemeur, R., Cnudde, J.P., Jacobs, P. (2004) Use of X-ray computed microtomography for non-invasive determination of wood anatomical characteristics. J. Struct. Biol. 148:11-21.

Sugimori, M., Lam, F. (1999) Macro-void distribution analysis in strand-based wood composites using an X-ray computer tomography technique. J. Wood Sci. 45:254-257.

Thoemen, H., Walther, T., Wiedmann, A. (2008) 3D simulation of macroscopic heat and mass transfer properties from the microstructure of wood fibre networks. Compos. Sci. Technol. 68:608-616.

van Houts, J., Bhattacharyya, D., Jayaraman, K. (2003) Viscoelastic behaviour of wood fibres during the hot pressing of medium density fiberboard. Holzforschung 57:391-399.

Walther, T. (2006) Methoden zur qualitativen und quantitativen Analyse der Mikrostruktur von Naturfaserwerkstoffen. PhD thesis, University of Hamburg, Hamburg, Germany.

Walther, T., Terzic, K., Donath, T., Meine, H., Beckmann, F., Thoemen, H. (2006) Microstructural analysis of lignocellulosic fiber networks. In: Developments in X-ray Tomography V, Ed. Bonse, U. Proc. SPIE Vol. 6318, 631812.

Wang, Y., Muszynski, L., Simonsen, J. (2007) Gold as an X-ray CT scanning contrast agent: effect on the mechanical properties of wood plastic composites. Holzforschung 61:723730.

Xie, Y., Feng, M., Deng, J. (2004) Quantification of UF and PF resins in MDF fiber with an X-ray fluorescence spectrometer. Wood Fiber Sci. 36:337-343.

Yamauchi, T. (2008) A method to determine lumen volume and collapse degree of pulp fibers by using bottleneck effect of mercury porosimetry. J. Wood Sci. 53:516-519.

Youngquist, J.A., Myers, G.C., Murmanis, L. (1987) Resin distribution in hardboard: evaluated by internal bond strength and fluorescence microscopy. Wood Fiber Sci. 19:215-224.

Received January 8, 2009. Accepted April 8, 2009. 